\title{
LINEAR CHEVALLEY ESTIMATES
}

\author{
TI WANG
}

\begin{abstract}
A Chevalley estimate for a germ of an analytic mapping $f$ is a function $l: \mathbb{N} \rightarrow \mathbb{N}$ such that if the composite with $f$ of a germ of an analytic function on the target vanishes to order at least $l(k)$, then it vanishes on the image to order at least $k$. Work of Izumi revealed the equivalence between regularity of a mapping (in the sense of Gabrielov, see $\S 1$ ) and the existence of a linear Chevalley estimate $l(k)$. Bierstone and Milman showed that uniformity of the Chevalley estimate is fundamental to several analytic and geometric problems on the images of mappings. The central topic of this article is uniformity of linear Chevalley estimates for regular mappings.

We first establish the equivalence between uniformity of a linear Chevalley estimate and uniformity of a "linear product estimate" on the image: A linear product estimate on a local analytic ring (or, equivalently, on a germ of an analytic space) means a bound on the order of vanishing of a product of elements which is linear with respect to the sum of the orders of its factors. We study the linear product estimate in the central case of a hypersurface (i.e., the zero set of an analytic function). Our results show that a linear product estimate is equivalent to an explicit estimate concerning resultants. In the special case of hypersurfaces of multiplicity 2 , this allows us to prove uniformity of linear product estimates.
\end{abstract}

\section{Notation.}

$\mathscr{O}_{X}:$ The structure sheaf on an analytic space $X$.

$\mathscr{O}_{X, \xi}$ : The fiber of the structure sheaf $\mathscr{O}_{X}$ at $\xi$, i.e., the local algebra of germs of analytic functions at $\xi \in X$.

in $(f(x))$ : The initial monomial in the Taylor expansion of $f(x) \in \mathbb{K} \llbracket x \rrbracket$ at 0 (cf. § 4).

$\exp (f(x))$ : The initial exponent of $f(x) \in \mathbb{K} \llbracket x \rrbracket$ (cf. Section 4).

$\nu_{\xi}(f)$ : The order of $f(x)$ with respect to the maximal ideal of $\mathscr{O}_{\xi}$, i.e., the maximal integer $k$ such that $f \in m_{\xi}^{k}$.

$\nu_{t}\left(w_{i, x}(t)\right)$ : The order of a root of a polynomial with respect to $t$ in a conic domain defined in $\S 3$.

$\operatorname{grk}(\phi)$ : The generic rank of an analytic mapping $\phi$.

$\operatorname{res}_{i}(P, f)(x)$ : The i-th resultant of polynomials $P(x, z)$ and $f(x, z)$, see $\S 3$.

$\sigma(q)$ : The order of $q(x)$ for a formal or convergent series.

$\tau(q)$ : The order of the remainder in the decomposition of $q(x)$, see $\S 4$.

$\mathscr{M}(X ; Z)$ : The ring of meromorphic functions on analytic spaces $X$ with all poles in analytic subspace $Z$.

Received by the editors April 30, 1994 and, in revised form, November 10, 1994.

1991 Mathematics Subject Classification. Primary 32S05, 32B10; Secondary 32S10, $13 \mathrm{C} 15$.

Key words and phrases. Chevalley estimates, Izumi's Theorem, irreducibility. 


\section{INTRODUCTION}

The subject of this article is the study of local invariants that distinguish the behavior of algebraic mappings from that of analytic mappings in general. Lojasiewicz [ $\llcorner O$ ] proved that semianalytic (or semialgebraic) sets lie in an analytic (or algebraic) subset of $\mathbb{R}^{n}$ of the same dimension. Therefore, the image of a polynomial map lies in an algebraic set of dimension equal to its rank, the generic rank of the Jacobian of the map (cf. [Mi, Theorem 1]). However, the example of Osgood (cf. Example 2.3) shows that, for a germ of an analytic mapping $y=\phi(x)$, the analogous condition (which was studied by Gabrielov in the early 1970 's, and which we call regularity) fails in general.

Let $\mathbb{K}=\mathbb{R}$ or $\mathbb{C}$. Consider a germ of an analytic mapping $y=\phi(x)$ over $\mathbb{K}$, where $x=\left(x_{1}, \ldots, x_{m}\right)$ and $y=\left(y_{1}, \ldots, y_{n}\right)$. Let $f(y)$ be a formal power series in $y-\phi(a)$. After being composed with $\phi, f(\phi(x))$ will be a formal power series in $x-a$. A linear estimate means that the order of vanishing of $f(\phi(x))$ in $x-a$ is linearly bounded from above by the order of vanishing of $f(y)$ in $y-\phi(a)$ modulo the ideal of formal relations among the components of $\phi(x)-\phi(a)$; we will call such an estimate a linear Chevalley estimate at $a$ (cf. the precise definitions in §2). Bierstone and Milman have shown that uniformity of Chevalley estimates is fundamental to several problems in both analysis and geometry of the images of analytic maps. Also, a uniform linear Chevalley estimate is essentially equivalent to a linear loss of differentiability in the solution of a classical problem on composite differentiable functions [BM4]. One of the main problems remaining from their work is uniformity of linear Chevalley estimates of regular mappings (cf. the conjecture in [BM4]); this is also the central topic of this paper.

Gabrielov proved the rank condition for analytic mappings (cf. [Ga]). A mapping (with a smooth source) is regular in the sense of Gabrielov at a given point if its generic rank at the point equals the minimal dimension of analytic closure of the image of a small neighborhood of this point. We say that a local analytic ring possesses a linear product estimate if the order of vanishing of a product is bounded by a linear function of the sum of the orders of factors. In the 1980's, Izumi established the equivalence among the following: linear product estimates on the target space, linear Chevalley estimates under the condition that the mapping is Gabrielov regular, and Gabrielov regularity of the mapping (cf. [I1], [I3]). Later, he showed that the linear product estimate on a given analytic local ring is equivalent to irreducibility of the germ of the analytic space [I2]. Rees [R1] extended the result to pseudovaluations on general local rings.

For a regular mapping, we prove that uniformity of the linear product estimate on the image of the mapping (in the sense of local rings of the image, see Definitions 2.5 and 2.8) and uniformity of the linear Chevalley estimate on the source space are equivalent:

Let $X$ and $Y$ be analytic spaces over $\mathbb{R}$ or $\mathbb{C}$ :

1.1. Theorem (Theorem 2.10). Let $\phi: Y \rightarrow X$ be an analytic mapping which is semiproper and regular. Then the following statements are equivalent:

1. The map $\phi$ admits a uniform linear Chevalley estimate on $Y$;

2. Let $Z=\phi(Y)$. There is a uniform linear product estimate on $Z$. 
We conjecture that uniformity of these linear estimates holds for all analytic mappings with the regularity condition. We can view a linear Chevalley estimate as a linear bound or orders of vanishing in an analytic equation $g=f(\phi)$, in terms of the order of a solution $f$ and that of a given function $g$. An analogous problem for a linear equation $g=A \cdot f$, where $A$ is a matrix with analytic functions as all its entries, concerns uniformity of the exponent in the ArtinRees estimate (lemma) (see [BM2], [BM3] and [W1]).

By Theorem 1.1, to study the uniformity of linear Chevalley estimates for regular mappings, it suffices to concentrate on the uniform linear product estimate on analytic subsets for irreducible components at every point. In this paper, we consider the case that the defining ideal of $X$ is principal. Then we can assume that near the point $(0,0), X$ is defined by the zero set of an irreducible and distinguished pseudopolynomial

$$
P(x, z)=z^{p}+\sum_{i=1}^{p} c_{i}(x) z^{p-i},
$$

where the $c_{i}(x)$ 's are analytic and $P(x, z)$ has order $p$ at $(x, z)=(0,0)$. Let $w_{1}(x), \ldots, w_{p}(x)$ be all the roots of $P(x, z)$. For any $f(x, z) \in \mathbb{C}\{x\}[z]$, we can define generalized resultants $\operatorname{res}_{i}(P, f)$ of $P(x, z)$ and $f(x, z)$ by the identity

$$
\prod_{i=1}^{p}\left(z-f\left(x, w_{i}(x)\right)\right)=z^{p}+\sum_{i=1}^{p} r e s_{i}(P, f)(x) z^{p-i} .
$$

By Puiseux's Theorem, we may add a parameter $t$ such that for fixed $x$, $P\left(t^{p !} x, z\right)$ has all the roots analytic in $t$. In a conic domain in the set where the discriminant of $P(x, z)$ never vanishes, we denote the roots of $P\left(t^{p !} x, z\right)$ by $w_{x, i}(t), i=1,2, \ldots, p$. Then we can define the order $\nu_{t}\left(w_{x, i}(t)\right)$ of each $w_{x, i}(t)$ in $t$ at $x=0$ (for details see $\S 3$ ). Denote by $\nu(f)$ the order of $f \in \mathcal{O}_{X, 0}$ with respect to the maximal ideal $m_{X, 0}$. The main theorem of $\S 3$ which is also one of the main new results of our work can then be stated as:

1.2. Theorem (Theorem 3.5). If $P(x, z)=z^{p}+\sum_{i=1}^{p} c_{i}(x) z^{p-i}$ is reduced and distinguished, then the following statements are equivalent:

(1) $P(x, z)$ is irreducible in $\mathscr{O}_{m+1}$.

(2) A linear product estimate holds for the germ $(X, 0)$, i.e., there are two constants $a \geq 1$ and $b \geq 0$ such that for all $f, g \in \mathscr{O}_{X, 0}$,

$$
\nu(f \cdot g) \leq a \cdot(\nu(f)+\nu(g))+b .
$$

(3) $P(x, z)$ has Property 1, i.e., there exist two constants $k_{1} \geq 1$ and $k_{2} \geq 0$ such that for all $f \in \mathcal{O}_{X, 0}$, we have

$$
\nu\left(\operatorname{res}_{p}(P, f)(x)\right) \leq k_{1} \cdot \nu(f)+k_{2} .
$$

(4) There exist two constants $l_{1} \geq 1$ and $l_{2} \geq 0$ such that for all $f \in \mathscr{O}_{X}, 0$,

$$
\frac{1}{p} \nu\left(\operatorname{res}_{p}(P, f)(x)\right) \leq l_{1} \cdot \min _{1 \leq i \leq p} \frac{1}{i} \nu\left(\operatorname{res}_{i}(P, f)(x)\right)+l_{2} .
$$

(5) There exist two constants $c_{1} \geq 1$ and $c_{2} \geq 0$ such that for all $f \in \mathscr{O}_{X, 0}$,

$$
\nu_{t} f\left(t^{p !} x, w_{x, i}(t)\right) \leq c_{1} \cdot \nu_{t} f\left(t^{p !} x, w_{x, j}(t)\right)+c_{2}
$$

for all $1 \leq i, j \leq p$. 
Remark. The relations among the constants in the above equivalences are explicit (cf. the remark after Theorem 3.5).

We study the uniform linear product estimates on hypersurfaces of multiplicity 2, i.e., $X$ has the multiplicity not more than 2 everywhere. Without loss of generality, we may assume that, locally, $X$ is given by a pseudopolynomial $P(x, z)=z^{2}+q(x)=0$ with the multiplicity of $q(x)$ not smaller than 2 . A detailed analysis of the irreducibility of $P(x, z)$ allows us to find a certain standard form for $q(x)$. Then we obtain

1.3. Theorem (Theorem 4.1). There is a uniform linear product estimate for hypersurfaces of multiplicity 2 .

By using the same idea, one can obtain uniformity of linear product estimates along the locus of multiplicity 3 in a hypersurface.

I would like to take this chance to express my sincere thanks to Professors E. Bierstone and P. Milman for their help. The formulation of the problem and some key techniques are due to them.

\section{Chevalley estimates: LINEARITY AND UNIFORMITY}

Let $\mathbb{K}=\mathbb{R}$ or $\mathbb{C}$. In this article, an analytic space $X$ over $\mathbb{K}$ is defined as follows: $X=\left(|X|, \mathscr{O}_{X}\right)$ is a local ringed space, where locally, there is an open set $U \subset \mathbb{K}^{n}$, a coherent ideal $I_{X} \subset \mathscr{O}_{U}$ such that $|X|$ is the support of $\mathscr{O}_{U} / I_{X}$, and $\mathscr{O}_{X}=\left.\left(\mathscr{O}_{U} / I_{X}\right)\right|_{|X|}$.

Let $X$ and $Y$ be analytic spaces over $\mathbb{K}$, and let $\phi: Y \rightarrow X$ be a morphism. The morphism $\phi$ induces a natural homomorphism of the structure sheaves

$$
\phi^{*}: \mathscr{O}_{X} \rightarrow \mathscr{O}_{Y} \text {. }
$$

For any $y \in Y, \phi^{*}$ determines a homomorphism of local rings

$$
\phi_{y}^{*}: \mathscr{O}_{X, \phi(y)} \rightarrow \mathscr{O}_{Y, y},
$$

and a homomorphism of the completions

$$
\hat{\phi}_{y}^{*}: \hat{\mathscr{O}}_{X, \phi(y)} \rightarrow \hat{\mathscr{O}}_{Y, y} .
$$

Let $m_{y}$ and $\hat{m}_{y}$ denote the maximal ideals of $\mathscr{O}_{Y, y}$ and $\hat{\mathscr{O}}_{Y, y}$ respectively, and let $R_{y}$ and $\hat{R}_{y}$ denote the kernels of $\phi_{y}^{*}$ and $\hat{\phi}_{y}^{*}$, respectively. For any $g \in \mathscr{O}_{X, \phi(y)}$, the lemma of Chevalley gives a comparison of orders of vanishing. The proof is elementary which can been found in [BM2].

2.1. Lemma. Let $y \in Y$. For each $k \in \mathbb{N}$, there exists $l \in \mathbb{N}$ such that if $g \in \hat{\mathscr{O}}_{X, \phi(y)}$, and $\hat{\phi}_{y}^{*}(g) \in m_{y}^{l+1}$, then $g \in R_{y}+m_{\phi(y)}^{k+1} \hat{\mathscr{O}}_{X, \phi(y)}$.

We will consider the following linear version of the Chevalley estimate:

2.2. Definition. There is a linear Chevalley estimate for $\phi$ at $y$ if there exist two constants $a \geq 1$ and $b \geq 0$ such that for all $g \in \mathscr{O}_{X, \phi(y)} / R_{y}$,

$$
\nu_{y}\left(\phi^{*}(g)\right) \leq a \cdot \nu_{\phi(y)}(g)+b,
$$

where $\nu_{y}$ and $\nu_{\phi(y)}$ are the orders with respect to the maximal ideals of $\mathscr{O}_{y}$ and $\mathscr{O}_{X, \phi(y)} / R_{y}$ respectively (see Notation). 
2.3. Example (Osgood). Let $\phi: \mathbb{K}^{2} \rightarrow \mathbb{K}^{3}$ be defined by

$$
\phi\left(x_{1}, x_{2}\right)=\left(x_{1}, x_{1} x_{2}, x_{1} x_{2} e^{x_{2}}\right) .
$$

In this case, $R_{0}=\{0\}$. Hence, $\mathscr{O}_{3,0} / R_{0}=\mathscr{O}_{3,0}$. We know that $g r k_{0}(\phi)=2$, $r_{2}(0)=r_{3}(0)=3$. Therefore, estimate (2.1) does not exist, either by a simple calculation or by Izumi's result. An analytic mapping has a linear Chevalley estimate at a point if and only if it is regular at the point [I3].

2.4. Theorem (Izumi) (cf. [I1]). Let $\phi:\left(\mathbb{K}^{n}, 0\right) \rightarrow\left(\mathbb{K}^{p}, 0\right)$ be the germ of analytic mapping of germs of regular spaces. Let $X \subset \mathbb{K}^{p}$ be defined by the irreducible ideal $\operatorname{ker}\left(\phi^{*}\right)$. Assume that $g r k_{0}(\phi)=\operatorname{dim}_{0} X$. Then the following statements are equivalent:

(1) $\phi$ has a linear Chevalley estimate, i.e. there exist two constants $a_{1} \geq 1$ and $b_{1} \geq 0$ such that for all $f \in \mathcal{O}_{X, 0}$,

$$
\nu\left(\phi^{*}(f)\right) \leq a_{1} \cdot \nu(f)+b_{1} .
$$

(2) $(X, 0)$ admits linear product estimates, i.e., there exist two constants $a_{2} \geq 1$ and $b_{2} \geq 0$ such that for all $f, g \in \mathscr{O}_{X, 0}$,

$$
\nu(f \cdot g) \leq a_{2} \cdot\{\nu(f)+\nu(g)\}+b_{2} .
$$

2.5. Definition. For a germ of analytic spaces $(X, \xi)$, it has a linear product estimate if (2.3) holds at $\xi$.

2.6. Theorem (Izumi's Theorem) (cf. [I2]; for the real case, see [R1]). Let $X$ be a germ of an analytic space. $X$ is irreducible if and only if $\mathscr{O}_{X}$ admits a linear product estimate.

Let $\phi: Y \rightarrow X$ be an analytic mapping.

\subsection{Definition.}

(1) An analytic mapping has a uniform Chevalley estimate if for any compact set $K \subset Y$ and any integer $k$, there is a function $l_{K}(k)$ such that for all $a \in K$, $l(k, a) \leq l_{K}(k)$.

(2) An analytic mapping has a uniform linear Chevalley estimate if for any compact subset $K \subset Y$, there exist two constants $a_{K} \geq 1$ and $b_{K} \geq 0$, by which we may define a linear function $l_{K}(k)=a_{K} \cdot k+b_{K}$ such that for all $a \in K, l(k, a) \leq l_{K}(k)$.

2.8. Definition. For a given set $X$, it has a uniform linear product estimate if for any compact set $K \subset X$, there are two constants $a_{K} \geq 1$ and $b_{K} \geq 0$ such that for any point $a \in K$, on any irreducible component $\tilde{X}_{a, j}$ of the germ of Zariski closure $\tilde{X}_{a}$ of the germ $X_{a}$, there is a linear product estimate (2.3) with $a_{2}=a_{K}$ and $b_{2}=b_{K}$.

2.9. Definition. Let $X$ and $Y$ be locally compact topological spaces, and let $f: Y \rightarrow X$ be a continuous mapping. Then $f$ is semiproper into $X$ if and only if for each compact subset $K \subset X$, there is a compact set (perhaps void) $L \subset Y$ such that

$$
f(L)=f(Y) \cap K
$$

Remark. A semiproper $\mathbb{C}$-analytic mapping is always Gabrielov regular, since its image is an analytic set (see [Wh, Theorem 11B]). 
In this section, the main result below shows that we can extend Izumi's result in a uniform way. The proof parallels that of Izumi with some technical preparations.

2.10. Theorem. Let $\phi: Y \rightarrow X$ be a semiproper and regular analytic mapping between analytic spaces. The mapping $\phi$ admits a uniform linear Chevalley estimate if and only if $\phi(Y)$ has a uniform product estimate.

By Hironaka's desingularization theorem (for a simpler proof, see [BM6]), we may assume that $Y$ is smooth. Theorem 2.10 follows from

2.11. Proposition. Let $B^{n} \subset \mathbb{K}^{n}$ be a closed ball. Let $\phi: \mathbb{K}^{n} \rightarrow X$ be a regular mapping of analytic spaces. Then, $\phi\left(B^{n}\right)$ has a uniform linear product estimate if and only if $\phi$ admits a uniform linear Chevalley estimate on $B^{n}$.

The proof is parallel to that of Izumi's theorem (Theorem 2.4). First, we state an algebraic lemma:

2.12. Lemma. Let $\Phi: A \rightarrow B$ be a finite and injective homomorphism between local Noetherian domains. Then there exists an integer $k>0$ such that, for every $f \in B$, there exist $g_{1}, \ldots, g_{k} \in A$, such that

$$
f^{k}+\sum_{i=1}^{k} \Phi\left(g_{i}\right) f^{k-i}=0
$$

Remark. Without loss of generality, we may assume that $A \subset B$ and $B$ is a finitely generated $A$-module:

$$
B=A\left(t_{1}, \ldots, t_{s}\right) \text {. }
$$

I am glad to thank Professor C. Huneke for telling me that we always have $k \leq s$.

Let $X$ be an analytic space over $\mathbb{K}$. The dimension of $X$ at a point $x$ means the Krull dimension of $\mathscr{O}_{X, x}$. An analytic mapping $\phi: X \rightarrow \mathbb{K}^{p}$ is said to be finite if for each point $x \in X, \mathscr{O}_{X}, x$ is a finitely generated module over $\mathcal{O}_{\mathbb{K}^{p}, \phi(x)}$ via the mapping $\phi_{x}^{*}$.

2.13. Corollary. Let $X$ be an analytic space of pure dimension $p$. Let $\Pi: X \rightarrow$ $\mathbb{K}^{p}$ be a finite mapping. Then there exist an integer $k>0$ and a neighborhood of $\alpha$, say $U \subset X$, such that for all $\beta \in U$ and any $f \in \mathscr{O}_{X, \beta}$, there exist $g_{1}, \ldots, g_{k} \in \mathscr{O}_{p, \Pi(\beta)}$,

$$
f^{k}+\sum_{i=1}^{k} g_{i}(\Pi) f^{k-i}=0
$$

Proof of Proposition 2.11. Suppose that $\phi$ admits a uniform linear Chevalley estimate on $B^{n}$. Our assumptions imply that for any compact subset $L \subset$ $\phi\left(B^{n}\right)$, there exists a compact subset $K \subset B^{n}$, such that for any $b \in L$ and any irreducible component of the germ of Zariski closure $\tilde{X}_{b}$ of the germ $L_{b}$ at $b$, say $\tilde{X}_{b, i}$, there exists a point $a \in K$ such that $\phi(a)=b$ and the mapping $\phi_{a}:\left(\mathbb{K}^{n}, a\right) \rightarrow \tilde{X}_{b, i}$ is regular. By our assumption, there exist $a_{K} \geq 1$ and $b_{K} \geq 0$ such that for any point $a \in K$, any $f \in \mathscr{O}_{\phi(a)}$,

$$
\nu_{a}(f(\phi)) \leq a_{K} \cdot \nu_{\phi(a)}(f)+b_{K} .
$$


For $f, g \in \mathscr{O}_{\phi(a), i}$, we have (denote by $\nu_{\phi(a), i}$ the valuation on $\tilde{X}_{b, i}$ )

$$
\begin{aligned}
\nu_{\phi(a), i}(f \cdot g) & \leq \nu_{a}(f(\phi) \cdot g(\phi)) \\
& =\nu_{a}(f(\phi))+\nu_{a}(g(\phi)) \\
& \leq a_{K}\left(\nu_{\phi(a)}(f)+\nu_{\phi(a)}(g)\right)+2 b_{K} \\
& \leq a_{K}\left(\nu_{\phi(a), i}(f)+\nu_{\phi(a), i}(g)\right)+2 b_{K}
\end{aligned}
$$

Thus, we have a uniform linear product estimate on $\phi\left(B^{n}\right)$.

On the other hand, assume that $\phi\left(B^{n}\right)$ admits a uniform linear product estimate. Let $\Pi: X \rightarrow \mathbb{K}^{p}$ be a finite mapping. Then, the mapping: $\Psi=$ $\Pi \circ \phi: \mathbb{K}^{n} \rightarrow \mathbb{K}^{p}$ has a generic rank $p$ since the regularity of $\Psi$ implies that

$$
\operatorname{Max} \operatorname{rank} \frac{\partial \Psi}{\partial\left(y_{1}, \ldots, y_{n}\right)}=p,
$$

where $\left(y_{1}, \ldots, y_{n}\right)$ are the coordinates on $\mathbb{K}^{n}$. It is known that there is a uniform linear Chevalley estimate for $\Psi$ (see [T1]). For this reason, we may assume that, for each compact subset $K \subset B^{n}$, there exists a constant $c_{K} \geq 1$ such that for any $f \in \mathscr{O}_{p, \Psi(a)}, \nu_{a}(f(\Psi)) \leq c_{K} \cdot \nu_{\Psi(a)}(f)$, for all $a$ in $K$. For a given compact subset $K \subset B^{n}$, the sets $L=\phi(K)$, and $\tilde{L}=\Pi(L)$ are compact. Let $a_{L}$ and $b_{L}$ be the coefficients in the uniform linear product estimate on $L$. We define a sequence of linear functions: Let $c=c_{K}$.

$$
e_{0}(\lambda)=c \lambda, \quad e_{p}(\lambda)=a_{L} \cdot c \cdot\left(\lambda+e_{p-1}(\lambda)\right)+b_{L} \cdot c \text { for } p=1,2, \ldots .
$$

We claim that: for any $a \in K$ and any $f \in \mathscr{O}_{\phi(a), i}$, if $\nu_{a}(f(\phi)) \geq e_{p-1}(\lambda)$, and if there exist $g_{1}, \ldots, g_{p} \in \mathscr{O}_{p}, \Psi(a)$, such that

$$
\nu_{\phi(a), i}\left(f^{p}+\sum_{i=1}^{p} g_{i}(\Pi) f^{p-i}\right) \geq e_{p-1}(\lambda),
$$

then, $\nu_{\phi(a), i}(f) \geq \lambda$.

The result follows from the claim: since the mapping $\Pi$ is finite, for any $f \in \mathscr{O}_{\phi(a), i}$, there exist $g_{1}, \ldots, g_{k} \in \mathscr{O}_{\Psi(a)}$ such that $f^{k}+\sum_{i} g_{i}(\Pi) f^{k-i}=0$. By Corollary 2.13 , the number $k$ is uniformly given on $\tilde{L}$. Thus, we may take the coefficients in $e_{k-1}$, i.e., $\nu_{a}(f(\phi)) \leq e_{k-1}\left(\nu_{\phi(a), i}(f)\right)$.

The proof of the claim is pointwise, which can be found in [I1].

\section{LINEAR PRODUCT ESTIMATES}

In this section, we consider a linear product estimate in the case where the defining ideal of the germ of $X$ is a principal ideal, i.e., $X$ is a hypersurface. In $\S \S 3.1$ and 3.2, we assume that the field is complex. The discussion of real cases is in $\$ 3.3$.

3.1. Generalized resultants. We assume that $X \subset \mathbb{C}^{m+1}$, and that at $0 \in X$ we have an irreducible and distinguished pseudopolynomial

$$
P(x, z)=z^{p}+\sum_{i=1}^{p} c_{i}(x) z^{p-i},
$$

where $x=\left(x_{1}, \ldots, x_{m}\right)$, such that in a neighborhood of 0 , the space $X=$ $\left\{(x, z) \in \mathbb{C}^{m+1}: P(x, z)=0\right\}$. 
After a linear coordinate change, we may further assume that for each $i$, $i=1, \ldots, p, \nu_{x}\left(c_{i}(x)\right) \geq i$. By the substitution $y=z+\frac{c_{1}(x)}{p}$, we reduce to the case that $c_{1}(x) \equiv 0$. form:

By the above assumptions, for any element $f \in \mathscr{O}_{X, 0}, f$ has a standard

$$
f=\sum_{i=1}^{p} a_{i}(x) z^{p-i}
$$

modulo the ideal. Moreover, the order of vanishing of $f$ in $\mathscr{O}_{X, 0}$ is given by

$$
\nu(f)=\min _{i}\left\{\nu\left(a_{i}(x)\right)+p-i\right\} .
$$

Let $w_{1}, \ldots, w_{p} \in \mathbb{F}$ be all the roots of $P(x, z)=0$. For any $f \in \mathbb{C}\{x\}[z]$, consider the polynomial

$$
\begin{aligned}
F(P, f)(x, y) & =\prod_{i=1}^{p}\left(y-f\left(x, w_{i}(x)\right)\right) \\
& =y^{p}+\sum_{i=1}^{p} \operatorname{res}_{i}(P, f)(x) \cdot y^{p-i} .
\end{aligned}
$$

It is easy to see that these coefficients $\operatorname{res}_{i}(P, f)(x)$ are polynomials of the coefficients of $P$ and $f$. Thus, $F(P, f)(x, y) \in \mathbb{C}\{x\}[y]$.

3.1. Definition. The coefficient $\operatorname{res}_{i}(P, f)(x)$ in (3.4) is called the $i$-th resultant of $P$ and $f$ for any polynomial $f$ in $\mathbb{C}\{x\}[z]$.

Remark.

1. By (3.4), for fixed $x$ and for each $k=1, \ldots, p$,

$$
\operatorname{res}_{k}(P, f)(x)=(-1)^{k} \sum_{1 \leq i_{1} \leq \ldots \leq i_{k} \leq p} f\left(x, w_{i_{1}}(x)\right) \ldots f\left(x, w_{i_{k}}(x)\right) .
$$

Each $\operatorname{res}_{k}(P, f)$ is a homogeneous polynomial in the coefficients of $f$ of degree $k$. In particular, $(-1)^{p} \operatorname{res}_{p}(P, f)(x)$ is the same as the conventional definition of the resultant of $P$ and $f$. For simplicity, we denote $(-1)^{p} \operatorname{res}_{p}(P, f)$ by $\operatorname{res}(P, f)$.

2. In the case where $x$ is a single variable, and if $P(x, z)$ is irreducible, by Puiseux's theorem, for any $f \in \mathbb{C}\{x\}[z]$ of degree less than $p$ :

$$
\frac{1}{p} \cdot \nu\left(\operatorname{res}_{p}(P, f)(x)\right)=\min _{j}\left(\frac{1}{j} \cdot \nu\left(\operatorname{res}_{j}(P, f)(x)\right)\right) .
$$

If $x$ is a multi-variable, (3.6) will no longer hold in general. However, by the following argument, we will see that the irreducibility of $P(x, z)$ is equivalent to a linear comparison between $\nu\left(\operatorname{res}_{p}(P, f)\right)$ and $\min _{i}\left(\nu\left(r e s_{i}(P, f)\right)\right)$. First, we define

Property 1. There are two constants $k_{1} \geq 1, k_{2} \geq 0$, such that for all $f \in \mathscr{O}_{X, 0}$,

$$
\nu(\operatorname{res}(P, f)) \leq k_{1} \nu(f)+k_{2} \text {. }
$$

Property 2. For any compact neighborhood $K$ of 0 , there exist two constants $k_{1} \geq 1$ and $k_{2} \geq 0$, such that for any point $\alpha \in K$, and for any irreducible 
component of the germ of $X_{\alpha}$, say $X_{\alpha, i}$ defined by $P_{\alpha, i}(x, z)=0$, and for any $f \in \mathscr{O}_{X_{n, i}}$,

$$
\nu\left(\operatorname{res}\left(P_{\alpha, i}, f\right)\right) \leq k_{1} \nu_{i, \alpha}(f)+k_{2},
$$

where $\nu_{i, \alpha}(f)$ denotes the order of $f$ in $\mathscr{O}_{X_{n}, i}$.

3.2. Proposition. Property 1 implies a linear product estimate for the germ $(X, 0)$. Further, if $X$ has Property 2 , then a uniform linear product estimate holds for any compact subset $K$ containing 0 .

Remark. In fact, Property 1 holds if and only if $(X, 0)$ admits a linear product estimate (see Theorem 3.5).

Let $I$ be the ideal generated by $P(x, z)$. Suppose that $f, g \in \mathscr{O}_{X, 0}$, and let $f=\sum_{i=1}^{p} a_{i}(x) z^{p-i}$, and $g=\sum_{i=1}^{p} b_{i}(x) z^{p-i}$. Then the product of $f$ and $g$ modulo $I$ is given by the Weierstrass division. Namely,

$$
f \cdot g(x, z)=\sum_{i=1}^{p} d_{i}(x) z^{p-i} \bmod (I),
$$

where the right side is the remainder in the division

$$
f \cdot g(x, z)=Q(x . z) \cdot P(x, z)+\sum_{i=1}^{p} d_{i}(x) z^{p-i}
$$

Therefore, $\nu(f \cdot g)=\min _{i}\left\{\nu_{x}\left(d_{i}(x)\right)+p-i\right\}$ as mentioned in (3.3).

Suppose that $P(x, z)$ is reduced, i.e., the discriminant $\Delta(x)$ of $P(x, z)$ is not zero. Therefore, in $K_{\Delta}=\{x: \Delta(x) \neq 0\}, P(x, z)$ has $p$ distinct solutions $w_{i}(x)$. Denote $t x=\left(t x_{1}, \ldots, t x_{n}\right)$. One can pick a point $\bar{x}$ and a neighborhood $U$ of $\bar{x}$ such that the conic neighborhood

$$
K_{U}=\left\{t^{p !} x: 0<|t| \leq 1, x \in U\right\}
$$

is a subset of $\{x: \Delta(x) \neq 0, \operatorname{in}(\Delta(x)) \neq 0\}$. We may further assume that all the coefficients of $P\left(t^{p !} x, z\right)$ are analytic in $K_{U}$. For fixed $y=\left(y_{1}, \ldots, y_{n}\right)$ in $K_{U}$, from Puiseux's theorem, we have an analytic decomposition in $t$ :

$$
P\left(t^{p !} y, z\right)=\prod_{i=1}^{p}\left(z-w_{y, i}(t)\right)
$$

where the $w_{y, i}(t)$ 's are analytic functions in $t$. We define $\nu_{t}$ as a mapping from $\mathbb{C}\{x\}\left[z_{1}, z_{2}, \ldots, z_{p}\right]$ to $\mathbb{N}:$ for any $f\left(x, z_{1}, z_{2}, \ldots, z_{p}\right) \in \mathbb{C}\{x\}\left[z_{1}, z_{2}, \ldots, z_{p}\right]$,

$$
\begin{aligned}
\nu_{t}(f) & =\nu_{t}\left(f\left(t^{p !} x, w_{x, 1}(t), \ldots, w_{x, p}(t)\right)\right. \\
& =\inf _{y \in K_{U}} \operatorname{ord}_{t}\left(f\left(t^{p !} y, w_{y, 1}(t), \ldots, w_{y, p}(t)\right)\right) .
\end{aligned}
$$

Clearly, if $h(x)$ is analytic in $x$, then $\nu_{t}\left(h\left(t^{p !} x\right)\right)=\operatorname{ord}(h(x)) \cdot p !$.

From the definition of $\nu_{t}$, we have a pseudovaluation on $\mathbb{C}\{x\}\left[z_{1}, z_{2}, \ldots, z_{p}\right]$. Recall that $\nu_{t}$ being a pseudovaluation means that for any $f, g$ in the ring,

$$
\nu_{t}(f+g) \geq \min \left\{\nu_{t}(f), \nu_{t}(g)\right\}, \quad \nu_{t}(f \cdot g) \geq \nu_{t}(f)+\nu_{t}(g) .
$$

3.3. Lemma. Let $f(x, z) \in \mathscr{O}_{X, 0}$. Then

$$
\nu(f(x, z)) \leq \frac{1}{p !} \nu_{t}\left(f\left(t^{p !} x, w_{x, i}(t)\right)\right)
$$

for each root $w_{x, i}(t)$ of $P\left(t^{p !} x, z\right)$. 
Proof. Consider the equation

$$
P\left(t^{p !} x, z\right)=z^{p}+\sum_{i=1}^{p} c_{i}\left(t^{p !} x\right) z^{p-i}=0 .
$$

Let $w_{i}(x)$ be a root of $P(x, z)$ in the sense given above. A root of $P\left(t^{p !} x, z\right)=$ 0 is denoted by $w_{x, i}(t)=w_{i}\left(t^{p !} x\right)$. It thus follows that

$$
p \cdot \nu_{t}\left(w_{x, i}(t)\right) \geq \min _{1 \leq j \leq p}\left(\nu_{t}\left(c_{j}\left(t^{p !} x\right)\right)+(p-j) \nu_{t}\left(w_{x, j}(t)\right)\right) .
$$

Therefore,

$$
\begin{aligned}
\nu_{t}\left(w_{x, i}(t)\right) & \geq \min _{1 \leq j \leq p} \frac{1}{j} \nu_{t}\left(c_{j}\left(t^{p !} x\right)\right) \\
& =\min _{1 \leq j \leq p} \frac{p !}{j} \nu_{x}\left(c_{j}(x)\right) \\
& \geq p !
\end{aligned}
$$

for each $i=1, \ldots, p$. For any $f(x, z) \in \mathscr{O}_{X, 0}$,

$$
\nu_{t} f\left(t^{p !} x, w_{x, i}(t)\right) \geq \nu_{x} f \cdot \min \left\{\nu_{t}\left(t^{p !} x\right), \nu_{t}\left(w_{x, i}(t)\right)\right\}=\nu_{x} f \cdot p ! .
$$

Thus, (3.11) follows.

For all $i, f \cdot g\left(t^{p !} x, w_{x, i}(t)\right)=\sum_{j=1}^{p} d_{j}\left(t^{p !} x\right) w_{x, i}(t)^{p-j}$. It yields an identity (3.13)

$$
\left(\begin{array}{c}
f \cdot g\left(t^{p !} x, w_{x, 1}(t)\right) \\
f \cdot g\left(t^{p !} x, w_{x, 2}(t)\right) \\
\vdots \\
f \cdot g\left(t^{p ! x}, w_{x, p}(t)\right)
\end{array}\right)=\left(\begin{array}{cccc}
1 & w_{x, 1}(t) & \ldots & w_{x, 1}(t)^{p-1} \\
1 & w_{x, 2}(t) & \ldots & w_{x, 2}(t)^{p-1} \\
\vdots & \vdots & \ddots & \vdots \\
1 & w_{x, p}(t) & \ldots & w_{x, p}(t)^{p-1}
\end{array}\right)\left(\begin{array}{c}
d_{p}\left(t^{p !} x\right) \\
d_{p-1}\left(t^{p !} x\right) \\
\vdots \\
d_{1}\left(t^{p !} x\right)
\end{array}\right) .
$$

Proof of Proposition 3.2. For a fixed point $x \in K_{U}$, let $A$ denote the coefficient matrix on the right side of the formula above. For all $k$,

$$
\nu_{t}\left(f \cdot g\left(t^{p !} x, w_{x, k}(t)\right)\right) \geq \min _{i}\left\{\nu_{t}\left(d_{i}\left(t^{p !} x\right)\right)+(p-i) \nu_{t}\left(w_{x, i}(t)\right)\right\} .
$$

For all $i=1, \ldots, p$, we have

$$
\nu_{t}\left(f \cdot g\left(t^{p !} x, w_{x, i}(t)\right)\right)=\nu_{t} f\left(t^{p !} x, w_{x, i}(t)\right)+\nu_{t} g\left(t^{p !} x, w_{x, i}(t)\right) .
$$

Since $f\left(t^{p !} x, w_{x, i}(t)\right)=\sum_{j} a_{j}\left(t^{p !} x\right) w_{x, i}(t)^{p-j}$, we get

$$
\left(\begin{array}{c}
f\left(t^{p !} x, w_{x, 1}(t)\right) \\
f\left(t^{p !} x, w_{x, 2}(t)\right) \\
\vdots \\
f\left(t^{p !} x, w_{x, p}(t)\right)
\end{array}\right)=A\left(\begin{array}{c}
a_{p}\left(t^{p !} x\right) \\
a_{p-1}\left(t^{p !} x\right) \\
\vdots \\
a_{1}\left(t^{p !} x\right)
\end{array}\right)
$$

where the matrix $A$ is defined in (3.13). Let $\Delta(x)$ denote the discriminant of $P(x, z)$. Then $\Delta\left(t^{p !} x\right)$ is the discriminant of $P\left(t^{p !} x, z\right)$ and clearly $|A|^{2}=$ 
$\Delta\left(t^{p}: x\right)$. Therefore, multiplying by the transposed matrix $A^{*}$ of cofactors of $A$, it follows

$$
A^{*}\left(\begin{array}{c}
f\left(t^{p !} x, w_{x, 1}(t)\right) \\
f\left(t^{p !} x, w_{x, 2}(t)\right) \\
\vdots \\
f\left(t^{p !} x, w_{x, p}(t)\right)
\end{array}\right)=|A|\left(\begin{array}{c}
a_{p}\left(t^{p !} x\right) \\
a_{p-1}\left(t^{p !} x\right) \\
\vdots \\
a_{1}\left(t^{p !} x\right)
\end{array}\right)
$$

From (3.17), obviously, we have for all $i$

$$
\frac{1}{2} \nu_{t} \Delta\left(t^{p !} x\right)+\nu_{t} a_{i}\left(t^{p !} x\right) \geq \min _{j}\left\{\nu_{t} f\left(t^{p !} x, w_{x, j}(t)\right)\right\} .
$$

Similarly, for $g$ and for all $i$,

$$
\frac{1}{2} \nu_{t} \Delta\left(t^{p !} x\right)+\nu_{t} b_{i}\left(t^{p !} x\right) \geq \min _{j}\left\{\nu_{t} g\left(t^{p !} x, w_{x, j}(t)\right)\right\} .
$$

Combining (3.18) with (3.19), we have for any $i$ and $k$,

$$
\begin{aligned}
& \nu_{t} \Delta\left(t^{p !} x\right)+\nu_{t} a_{i}\left(t^{p !} x\right)+\nu_{t} b_{k}\left(t^{p !} x\right) \\
& \quad \geq \min _{j}\left\{\nu_{t} f\left(t^{p !} x, w_{x, j}(t)\right)\right\}+\min _{j}\left\{\nu_{t} g\left(t^{p !} x, w_{x, j}(t)\right)\right\} .
\end{aligned}
$$

Using Lemma 3.3 and Property 1 , we obtain for any $j, k$,

$$
\begin{aligned}
\nu_{t}\left(f\left(t^{p !} x, w_{x, j}(t)\right)\right) & \leq \nu_{t}\left(\prod_{i=1}^{p}\left(f\left(t^{p !} x, w_{x, i}(t)\right)\right)\right) \\
& =\nu_{t}\left(\operatorname{res}(P, f)\left(t^{p !} x\right)\right) \\
& \leq p !\left(k_{1} \nu(f)+k_{2}\right) \leq p !\left(k_{1} \frac{\nu_{t}\left(f\left(t^{p !} x, w_{x, k}(t)\right)\right)}{p !}+k_{2}\right) .
\end{aligned}
$$

Thus, there are two constants $c_{1} \geq 1$ and $c_{2} \geq 0$, where $c_{1}=k_{1}$ and $c_{2}=$ $p ! k_{2}$, such that for any $f \in \mathscr{O}_{X, 0}$, and any $j$ and $k, \nu_{t} f\left(t^{p !} x, w_{x, j}(t)\right) \leq$ $c_{1} \nu_{t} f\left(t^{p !} x, w_{x, k}(t)\right)+c_{2}$. Here, (3.14), (3.15), (3.20) and (3.21) imply

$$
\begin{aligned}
& \nu_{t} \Delta\left(t^{p !} x\right)+\nu_{t} a_{i}\left(t^{p !} x\right)+\nu_{t} b_{j}\left(t^{p !} x\right) \\
& \quad \geq \min _{j}\left\{\nu_{t} f\left(t^{p !} x, w_{x, j}(t)\right)\right\}+\min _{j}\left\{\nu_{t} g\left(t^{p !} x, w_{x, j}(t)\right)\right\} \\
& \quad \geq \frac{1}{c_{1}} \min _{k}\left\{\nu_{t}\left(d_{k}\left(t^{p !} x\right)\right)+(p-k) p !\right\}-\frac{c_{2}}{c_{1}},
\end{aligned}
$$

where we assume that

$$
\nu_{t}\left(g\left(t^{p !} x, w_{x, l}(t)\right)\right)=\min _{i}\left\{\nu_{t}\left(g\left(t^{p !} x, w_{x, i}(t)\right)\right)\right\} .
$$

Clearly, since each $\Delta(x), a_{i}(x), b_{j}(x)$ and $d_{k}(x)$ are analytic with respect to $x$, we have

$$
\begin{aligned}
& \nu_{t} \Delta\left(t^{p !} x\right)=p ! \cdot \nu_{x} \Delta(x) ; \quad \nu_{t} a_{i}\left(t^{p !} x\right)=p ! \cdot \nu\left(a_{i}(x)\right) ; \\
& \nu_{t} b_{j}\left(t^{p !} x\right)=p ! \cdot \nu\left(b_{j}(x)\right) ; \quad \nu_{t} d_{k}\left(t^{p !} x\right)=p ! \cdot \nu\left(d_{k}(x)\right) \text {. }
\end{aligned}
$$


It is therefore

$$
\nu \Delta(x)+\nu a_{i}(x)+\nu b_{j}(x) \geq \frac{1}{c_{1}} \min _{k}\left\{\nu\left(d_{k}(x)\right)+p-k\right\}-\frac{c_{2}}{c_{1} p !} .
$$

By (3.3) and choosing proper $i$ and $j$, we may have

$$
\nu \Delta(x)+\nu a_{i}(x)+\nu b_{j}(x) \leq \nu \Delta(x)+\nu(f)+\nu(g) .
$$

Then, we obtained

$$
\nu(f \cdot g) \leq k_{1}(\nu(f)+\nu(g))+k_{1} \nu(\Delta(x))+k_{2},
$$

where $k_{1}$ and $k_{2}$ are exactly the same as in Property 1 . Hence, it is a linear product estimate as required, and it gives a uniform linear product on $X$ if Property 2 holds.

\subsection{Criteria for linear product estimates.}

3.4. Proposition. Assume that $P(x, z)$ is reduced. Then for all $f$, we have

$$
\nu(f) \leq \min _{1 \leq i \leq p} \frac{\nu\left(\operatorname{res}_{i}(P, f)\right)}{i} \leq \nu(f)+\frac{1}{2} \nu(\Delta(x)) .
$$

Proof. For all $i=1, \ldots, p$, for a fixed point $x \in K_{U}$,

$$
\left(f\left(t^{p !} x, w_{x, i}(t)\right)\right)^{p}+\sum_{i=1}^{p} r e s_{k}(P, f)\left(t^{p !} x\right)\left(f\left(t^{p !} x, w_{x, i}(t)\right)\right)^{p-k} \equiv 0 .
$$

Therefore,

$p \cdot \nu_{t}\left(f\left(t^{p !} x, w_{x, i}(t)\right)\right) \geq \min _{k}\left\{\nu_{t}\left(r e s_{k}(P, f)\left(t^{p !} x\right)\right)+(p-k) \cdot \nu_{t}\left(f\left(t^{p !} x, w_{x, i}(t)\right)\right)\right\}$,

i.e., there exists some $k$ ( $k$ may depend on $i$ ) such that

$$
\nu_{t}\left(f\left(t^{p !} x, w_{x, i}(t)\right)\right) \geq \frac{1}{k} \nu_{t}\left(\operatorname{res}_{k}(P, f)\left(t^{p !} x\right)\right) .
$$

Thus,

$$
\min _{i} \nu_{t}\left(f\left(t^{p !} x, w_{x, i}(t)\right)\right) \geq \min _{i}\left\{\frac{1}{i} \nu_{t}\left(\operatorname{res}_{i}(P, f)\left(t^{p !} x\right)\right)\right\} .
$$

By applying (3.18), for all $j=1, \ldots, p$,

$$
\frac{1}{2} \nu_{t} \Delta\left(t^{p !} x\right)+\nu_{t} a_{j}\left(t^{p !} x\right) \geq \min _{i}\left\{\frac{1}{i} \nu_{t}\left(\text { res }_{i}(P, f)\left(t^{p !} x\right)\right)\right\}
$$

Again, since all the entries above are analytic in $x$, the second half of (3.26) follows from (3.3) and (3.23) as required. The first half of (3.26), i.e., $\nu(f) \leq$ $\min _{1 \leq i \leq p} \nu\left(r e s_{i}(P, f)\right) / i$, is an immediate consequence of Lemma 3.3 and formula (3.5).

3.5. Theorem. If $P(x, z)=z^{p}+\sum_{i=1}^{p} c_{i}(x) z^{p-i}$ is reduced and distinguished, then the following statements are equivalent:

(1) $P(x, z)$ is irreducible in $\mathscr{O}_{m+1}$.

(2) A linear product estimate holds for the germ $(X, 0)$, i.e., there are two constants $a \geq 1$ and $b \geq 0$ such that for all $f, g \in \mathscr{O}_{X, 0}$,

$$
\nu(f \cdot g) \leq a \cdot(\nu(f)+\nu(g))+b .
$$


(3) $P(x, z)$ has Property 1, i.e., there exist two constants $k_{1} \geq 1$ and $k_{2} \geq 0$ such that for all $f \in \mathscr{O}_{X}, 0$, we have

$$
\nu\left(\operatorname{res}_{p}(P, f)(x)\right) \leq k_{1} \cdot \nu(f)+k_{2} .
$$

(4) There exist constants $l_{1} \geq 1$ and $l_{2} \geq 0$ such that for all $f \in \mathscr{O}_{X, 0}$,

$$
\frac{1}{p} \nu\left(\operatorname{res}_{p}(P, f)(x)\right) \leq l_{1} \cdot \min _{1 \leq i \leq p} \frac{1}{i} \nu\left(\operatorname{res}_{i}(P, f)(x)\right)+l_{2} .
$$

(5) There exist constants $c_{1} \geq 1$ and $c_{2} \geq 0$ such that for all $f \in \mathscr{O}_{X}, 0$,

$$
\nu_{t} f\left(t^{p !} x, w_{x, i}(t)\right) \leq c_{1} \cdot \nu_{t} f\left(t^{p !} x, w_{x, j}(t)\right)+c_{2},
$$

for all $1 \leq i, j \leq p$.

Proof.

(1) $\Rightarrow$ (2): By Izumi's theorem (see Theorem 2.4).

$(2) \Rightarrow(1)$ : If $P(x, z)=f(x, z) \cdot g(x, z)$, it is then apparent that (2) can not hold.

$(2) \Rightarrow(3)$ : We define a sequence of linear functions

$$
e_{1}(\lambda)=\lambda ; \quad \text { for all } q \geq 1, \quad e_{q+1}(\lambda)=a \cdot\left(\lambda+e_{q}(\lambda)\right)+b .
$$

Using Tougeron's idea (cf. [I1]), we can claim that for a given $f \in \mathscr{O}_{X, 0}$, if there exist $g_{i}(x) \in \mathscr{O}_{m}$ such that

$$
\nu\left(f^{q}(x, z)+\sum_{j=1}^{q} g_{j}(x) f^{q-j}(x, z)\right) \geq e_{q}(\lambda)
$$

and $\nu_{t} f\left(t^{p !} x, w_{x, i}(t)\right) \geq p ! e_{q}(\lambda)$, then we have $\nu(f) \geq \lambda$. Our claim implies that there are two constants $b_{1} \geq 1$ and $b_{2} \geq 0$ such that for all $i=1, \ldots, p$,

$$
\nu_{t}\left(f\left(t^{p !} x, w_{x, i}(t)\right)\right) \leq b_{1} \cdot \nu(f)+b_{2} .
$$

Indeed, since

$$
f^{p}(x, z)+\sum_{i=1}^{p} r e s_{i}(x) f^{p-i}(x, z)=0,
$$

we can take $b_{1} \lambda+b_{2}=p ! e_{p}(\lambda)$. But, it is apparent that (3.32) implies (3) with $k_{i}=b_{i} /(p-1) !, i=1,2$.

(3) $\Rightarrow(4)$ : For all $i$,

$$
\begin{aligned}
\frac{1}{p} \nu\left(\operatorname{res}_{p}(P, f)(x)\right) & \leq \frac{k_{1}}{p} \nu(f)+\frac{k_{2}}{p} \\
& \leq \frac{k_{1}}{i \cdot p} \nu\left(\operatorname{res}_{i}(P, f)(x)\right)+\frac{k_{2}}{p} .
\end{aligned}
$$

Hence, (4) follows.

$(4) \Rightarrow(5)$ : For a given $f \in \mathcal{O}_{X, 0}$, by (4) we have: for any $1 \leq i, j \leq p$,

$$
\nu_{t}\left(\operatorname{res}_{p}(P, f)\left(t^{p !} x\right)\right) \leq l_{1} \cdot p \cdot \min _{i}\left\{\frac{1}{i} \nu_{t}\left(\operatorname{res}_{i}(P, f)\left(t^{p !} x\right)\right)\right\}+l_{2} \cdot p \cdot p ! .
$$


Using Proposition 3.4, there are constants $l_{1}^{\prime} \geq 1$ and $l_{2}^{\prime} \geq 0$ such that

$$
\begin{aligned}
\nu_{t}\left(f\left(t^{p !} x, w_{x, i}(t)\right)\right) & \leq \nu_{t}\left(\operatorname{res}_{p}(P, f)\left(t^{p !} x\right)\right) \\
& \leq l_{1} \cdot p \cdot \min _{i}\left\{\frac{1}{i} \nu_{t}\left(\operatorname{res}_{i}(P, f)\left(t^{p !} x\right)\right)\right\}+l_{2} \cdot p \cdot p ! \\
& =\left(l_{1} \cdot p \cdot \min \left\{\frac{1}{i} \nu\left(\operatorname{res}_{i}(P, f)(x)\right)\right\}+l_{2} \cdot p\right) \cdot p ! \\
& \leq\left(l_{1} \cdot p \cdot\left(\nu(f)+\frac{1}{2} \nu(\Delta(x))\right)+l_{2} \cdot p\right) \cdot p ! .
\end{aligned}
$$

By Lemma 3.3, (3.34) gives

$$
\begin{aligned}
\nu_{t}\left(f\left(t^{p !} x, w_{x, i}(t)\right)\right) & \leq \nu_{t}\left(\operatorname{res}_{p}(P, f)\left(t^{p !} x\right)\right) \\
& \leq l_{1} \cdot p \cdot\left(\nu_{t}\left(f\left(t^{p !} x, w_{x, j}(t)\right)\right)\right)+l_{2} \cdot p \cdot p !,
\end{aligned}
$$

as required.

$(5) \Rightarrow(2)$ : For any $f, g \in \mathcal{O}_{X, 0}, f=\sum_{i} a_{i}(x) z^{p-i}$ and $g=\sum_{j} b_{j}(x) z^{p-j}$, we have (see (3.22))

$$
\nu_{t} \Delta\left(t^{p !} x\right)+\nu_{t} a_{i}\left(t^{p !} x\right)+\nu_{t} b_{j}\left(t^{p !} x\right) \geq \frac{1}{c_{1}} \min _{k}\left\{\nu_{t}\left(d_{k}\left(t^{p !} x\right)\right)+(p-k) \cdot p !\right\}-\frac{c_{2}}{c_{1}}
$$

for any $i, j=1, \ldots, p$. Therefore, by (3.24),

$$
\frac{1}{c_{1}} \nu(f \cdot g)-\frac{c_{2}}{c_{1} \cdot p !} \leq \nu(\Delta(x))+\nu\left(a_{i}(x)\right)+\nu\left(b_{j}(x)\right) \leq \nu(\Delta(x))+\nu(f)+\nu(g) .
$$

It follows that

$$
\nu(f \cdot g) \leq c_{1}(\nu(f)+\nu(g))+c_{1} \nu \Delta(x)+\frac{c_{2}}{p !}
$$

as required.

Remark. We just showed a necessary circle of a logical proof of the theorem as above. We list some relations among all the coefficients above (for the proof, refer to [W2]): $k_{2}$.

1. When (2) is derived from (3), we may take $a=k_{1}$ and $b=k_{1} \cdot \nu(\Delta(x))+$

2. (2) $\Rightarrow(3)$ : we may take $k_{1}$ and $k_{2}$ as $k_{1} \lambda+k_{2}=p \cdot e_{p}(\lambda)$, where $e_{1}(\lambda)=\lambda$, and $e_{q+1}(\lambda)=a \cdot\left(\lambda+e_{q}(\lambda)\right)+b$, for $q=1,2, \ldots$.

3. (3) $\Rightarrow(4)$ : we may take $l_{1}=\frac{k_{1}}{p}$ and $l_{2}=\frac{k_{2}}{p}$.

4. (4) $\Rightarrow$ (3): we may take $k_{1}=p \cdot l_{1}$ and $k_{2}=p \cdot\left[\frac{1}{2} l_{1} \nu(\Delta(x))+l_{2}\right]$.

5. (3) $\Rightarrow$ (5): we may take $c_{1}=k_{1}$ and $c_{2}=p ! k_{2}$.

6. (5) $\Rightarrow(2)$ : we may take $a=c_{1}$ and $b=c_{1} \cdot \nu(\Delta(x))+\frac{c_{2}}{p !}$.

7. (4) $\Rightarrow(5)$ : we may take $c_{1}=l_{1} \cdot p$ and $c_{2}=\frac{1}{2} l_{1} \cdot p \cdot p ! \cdot \nu(\Delta(x))+l_{2} \cdot p \cdot p$ !.

8. (5) $\Rightarrow(4)$ : we may take $l_{1}=c_{1}$ and $l_{2}=\frac{c_{2}}{p !}$.

Remark. Clearly, as a consequence of the explicit nature of our estimates, we have the version of a uniform linear comparison of Theorem 3.5, namely, for each (2), (3), (4) and (5), if the coefficients exist on any compact subset of the analytic space, i.e., for any irreducible component at any point in the compact set, we have one of (2), (3), or (4) for the factor of $P(x, z)$, then a uniform linear product estimate exists. 
3.3. Real cases. In the discussion of linear product estimates on hypersurfaces in $\S \S 3.1$ and 3.2, we assume that the field is complex. Now let us examine the case of real hypersurfaces. First, we give some elementary facts:

Let $f(y)=\sum_{\alpha} a_{\alpha} y^{\alpha} \in \mathbb{C} \llbracket y \rrbracket$. We write $\overline{f(y)}=\sum_{\alpha} \bar{a}_{\alpha} y^{\alpha}$, where $\bar{a}_{\alpha}$ denotes the conjugate of $a_{\alpha}$. Then, we have

3.6. Lemma. Let $f(y)$ and $g(y) \in \mathbb{C} \llbracket y \rrbracket$. Then,

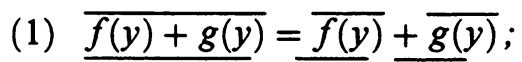

(2) $\overline{f(y) \cdot g(y)}=\overline{f(y)} \cdot \overline{g(y)}$;

(3) $\overline{f(y)}=f(y)$ if and only if $f(y) \in \mathbb{R} \llbracket y \rrbracket$.

3.7. Lemma. Suppose that $F(y)$ is an irreducible real formal power series. If $F(y)$ is reducible in $\mathbb{C} \llbracket y \rrbracket$, then it has a unique decomposition in the form

$$
F(y)=G(y) \cdot \overline{G(y)},
$$

where $G(y)$ is irreducible in $\mathbb{C} \llbracket y \rrbracket$.

It is easy to prove the two lemmas above.

Let $P(x, z)=z^{p}+\sum_{i} c_{i}(x) z^{p-i}$ be an irreducible real pseudopolynomial. Suppose that $P(x, z)$ is reducible in $\mathbb{C} \llbracket x \rrbracket[z]$. By Lemma 3.7, we can assume that $P(x, z)=Q(x, z) \cdot \overline{Q(x, z)}$. For any $f(x, z) \in \mathbb{C} \llbracket x \rrbracket[z]$, the resultant $\operatorname{res}_{p}(P, f)(x)$ is therefore the product $\operatorname{res}_{q}(Q, f)(x) \cdot \operatorname{res}_{q}(Q, f)(x)$. With this notation, we can show

\subsection{Lemma.}

$$
\operatorname{res}_{q}(Q, f)(x)=\overline{\operatorname{res}(\bar{Q}, \bar{f})} .
$$

As the consequence, assuming that $f(x, z)$ is a real pseudopolynomial, we have

$$
\operatorname{res}_{p}(P, f)(x)=\left(\operatorname{res}_{q}(\bar{Q}, f)(x)\right) \cdot\left(\overline{\operatorname{res}_{q}(\bar{Q}, f)(x)}\right) .
$$

Suppose that $X$ is a real hypersurface defined by a real analytic function. We consider on an irreducible component of $X$ at $(0,0)$ (for simplicity, still denoted by $X$ ) which is given by the germ of real zero set of an irreducible pseudopolynomial

$$
P(x, z)=z^{p}+\sum_{i=1}^{p} c_{i}(x) z^{p-i}=0,
$$

where $c_{i}(x) \in \mathbb{R}\{x\}$. We denote by $X^{\mathbb{C}}$ the complexification of $X$ defined as the zero set of $P(x, z)$ in $\mathbb{C}^{n+1}$. By Lemma 3.7, $X^{\mathrm{C}}$ is either irreducible, or it can be decomposed into a pair of conjugates $X^{\prime}$ and $\bar{X}^{\prime}$ defined by two irreducible factors of $P(x, z)=Q(x, z) \cdot \bar{Q}(x, z)$, respectively.

3.9. Lemma. Let $Q(x, z) \in \mathbb{C}\{x\} \llbracket z \rrbracket$ be an irreducible polynomial. The linear product estimate on the germ $X^{\prime}$ of the analytic space defined by $Q(x, z)=0$ is the same as that on the germ $\bar{X}^{\prime}$ defined by $\bar{Q}(x, z)=0$.

Proof. This is a consequence of Lemma 3.8 and Theorem 3.5.

Now, the following result can be verified easily: 
3.10. Proposition. For a real analytic space $X$, it has a uniform linear product estimate if there is a uniform linear product estimate on its complexification.

\section{A UNIFORM LINEAR PRODUCT ESTIMATE FOR HYPERSURFACES OF MULTIPLICITY 2}

Let $f(x)=\sum_{\alpha} a_{\alpha} x^{\alpha} \in \mathbb{K} \llbracket x \rrbracket$, where $x=x_{1}, \ldots, x_{n}$ and $\alpha \in \mathbb{N}^{n}$ ( $\mathbb{N}$ is the set of non-negative integers). The support of $f(x)$ is the set $\left(\alpha \in \mathbb{N}^{n} \mid a_{\alpha} \neq 0\right)$. Define an order on $\mathbb{N}^{n}$ by ordering each $\alpha \in \mathbb{N}^{n}$ by the lexicographic order on $(n+1)$-tuple $(|\alpha|, \alpha)$. This is a linear order on $\mathbb{N}^{n}$. By the initial monomial of $f$ we mean the monomial $x^{\alpha^{\prime}}$ such that $\alpha^{\prime}$ is the smallest element in the support of $f(x)$. Moreover, $\alpha^{\prime}$ is the exponent of $f(x)$.

4.1. Normal forms. Let $X$ be the complex space defined by the zero set of an irreducible pseudopolynomial of order 2: $P(x, z)=z^{2}+p(x) z+q(x)=0$, where we may assume that $p(x) \equiv 0$ and $\nu(q(x)) \geq 2$. It is evident that $P(x, z)$ is irreducible if and only if $q(x)$ is not a completely squared element in the ring $\mathbb{C}\{x\}$ (i.e., there is no $h(x) \in \mathbb{C}\{x\}$ such that $q(x)=h^{2}(x)$ ).

\subsection{Theorem. There is a uniform linear product estimate for $X$.}

We first show a linear product estimate for $X$ at $0 \in X$ with explicit formulas for the coefficients.

4.2. Lemma. Let $q(x) \in \mathbb{C}\{x\}$. Assume that there are two sequences $s_{i}(x)$ and $t_{i}(x)$ in $\mathbb{C} \llbracket x \rrbracket$ such that for each $i, q(x)=s_{i}^{2}(x)+t_{i}(x)$ and $\lim _{i \rightarrow \infty} \nu\left(t_{i}(x)\right)=$ $\infty$. Then, $q(x)$ is a completely squared element in $\mathbb{C}\{x\}$.

Proof. We can assume that each $s_{i}(x)$ has the same initial term as $q(x)$, i.e., the same first term in their Taylor expansions. Then the sequence $\left\{s_{i}(x)\right\}$ is a Cauchy sequence in $\mathbb{C}\{x\}$. Thus, it converges to an element $s(x) \in \mathbb{C} \llbracket x \rrbracket$ since $\mathbb{C} \llbracket x \rrbracket$ is complete. It follows from the Hensel Lemma (cf. [Na, 44.3]) or Artin's approximation theorem that $s(x) \in \mathbb{C}\{x\}$.

4.3. Definition. Let $q(x) \in \mathbb{C}\{x\}$. We define a decomposition of $q(x)$ :

$$
q(x)=s^{2}(x)+t(x)
$$

with the properties:

D-1. $s(x)$ and $t(x)$ are in $\mathbb{C} \llbracket x \rrbracket$;

D-2. $t(x)=q(x)$, or $\nu(t(x))>\nu(q(x))$;

D-3. $\nu(t(x))$ is the maximal in all such possible decompositions.

Remark.

1. Lemma 4.2 implies that this definition is well-defined, i.e., we always have a decomposition as described above.

2. We denote $\sigma(q)=\nu\left(s^{2}(x)\right)$ and $\tau(q)=\nu(t(x))$. Apparently, $\sigma(q)$ and $\tau(q)$ are uniquely determined by $q$. However, in general, the decompositions of $q(x)$ satisfying (D-1), (D-2) and (D-3) are not unique (for instance, we can add a higher-order term to $s(x)$ and $t(x)$ if both are not zero).

3. $q(x)$ is a completely squared element in $\mathbb{C}\{x\}$ if and only if $t(x)=0$. If $s(x) \neq 0, \exp \{q(x)\}=\exp \left\{\left(s^{2}(x)\right\}\right.$ and $\exp \{t(x)\}>\exp \{q(x)\}$. For our purpose, we will not consider the case $t(x)=0$ or $s(x)=0$ in $\S 4.1$. So, we assume $s(x) \neq 0$ and $t(x) \neq 0$ throughout these sections. 
4. If $f(x)$ and $g(x)$ are equivalent, i.e., $f(x)=g(x) h(x)$ for some invertible $h(x)$ in $\mathbb{C}\{x\}$, then, $\sigma(f(x))=\sigma(g(x))$ and $\tau(f(x))=\tau(g(x))$.

5. By Lemma 4.2, we may define a decomposition of $q(x)$ :

$$
q(x)=s^{2}(x)+\bar{t}(x)
$$

with the properties of (D-2) and (D-3), plus that both $\bar{s}$ and $\bar{t}$ are analytic. Then, it is easy to check that $\nu(s(x))=\nu(\bar{s}(x))$ and $\nu(t(x))=\nu(\bar{t}(x))$. In fact, it is clear that $\nu(\bar{t}(x)) \leq \nu(t(x))$. On the other hand, suppose we have (1): $q(x)=s^{2}(x)+t(x)$. If $s(x)$ is not analytic, and $t(x) \neq 0$. Then, obviously, by taking a high jet of $s(x)$ (for instance, higher than the order of $t(x)$ ), we will have a convergent decomposition without changing the order of $t(x)$. That means $\nu(\bar{t}(x)) \geq \nu(t(x))$.

Clearly, in a given coordinate system, among all the possible decompositions (4.1), $\exp (t(x))$ has the maximum.

4.4. Definition. We call (4.1) a proper decomposition in a coordinate system if $\exp (t(x))$ reaches its maximum.

Of course, in general, such a decomposition is not unique.

4.5. Lemma. Let $q(x)=s^{2}(x)+t(x)$ be a decomposition, where $s(x) \neq 0$ and $t(x) \neq 0$. Let in $(s(x))=s_{0} x^{\alpha}$, and in $(t(x))=t_{0} x^{\beta}$. Then, it is a proper decomposition of $q(x)$ if and only if $\alpha \nprec \beta$, i.e.,

$$
x^{\alpha} \nmid x^{\beta} \text {. }
$$

Proof. Suppose that (4.2) is false. It means that $t(x)=t_{0} x^{\alpha+\gamma}+h(x)$ for some $0 \neq \gamma \in \mathbb{N}^{m}$. Let $s(x)=s_{0} x^{\alpha}+g(x)$. Then,

$$
\begin{aligned}
q(x) & =\left(s_{0} x^{\alpha}+g(x)\right)^{2}+t_{0} x^{\alpha+\gamma}+h(x) \\
& =\left(s_{0} x^{\alpha}+g(x)+\frac{t_{0} x^{\gamma}}{2 s_{0}}\right)^{2}+h(x)-\frac{t_{0} g(x) x^{\gamma}}{s_{0}}-\frac{t_{0}^{2} x^{2 \gamma}}{4 s_{0}^{2}} .
\end{aligned}
$$

It is clear that $\exp (h(x))>\exp (t(x)), \exp \left(g(x) x^{\gamma}\right)>\exp (t(x))$ and $\exp \left(x^{2 \gamma}\right)>$ $\exp (t(x))$, which contradicts the statement that $q(x)=s^{2}(x)+t(x)$ is a proper decomposition of $q(x)$.

It is then easy to check that (4.2) is also a sufficient condition.

Let $q(x)$ be the same as described above. We assume that $s(x) \neq 0$ and $t(x) \neq 0$ in the decomposition (4.1) of $q(x)$.

4.6. Lemma. There exists a suitable linear coordinate change: $x \rightarrow(y, \bar{x})$, such that under this coordinate,

$$
q(x)=\left(\left(y^{k}+g(y, \bar{x})\right)^{2}+h(y, \bar{x})\right) \cdot i(x)
$$

with the following properties:

$\mathrm{N}-1 . \quad i(x)$ is invertible in $\mathbb{C} \llbracket x \rrbracket$;

$\mathrm{N}-2 . \quad g(y, \bar{x})$ and $h(y, \bar{x})$ are both pseudopolynomials in $y$. Moreover, $\operatorname{deg}_{y}\{g\}<k$, and $\operatorname{deg}_{y}\{h\}<k$

N-3. $\nu(g) \geq k ; \nu(h) \geq 2 k$.

4.7. Definition. A decomposition (4.3) of $q(x)$ satisfying (N-1), (N-2) and $(\mathrm{N}-3)$ is called a normal decomposition of $q(x)$. 
By Lemma 4.5, a normal decomposition must be a proper decomposition.

Let $f(y, \bar{x})=\left(y^{k}+m(y, \bar{x})\right)^{2}+n(y, \bar{x})$, where

P-1. $m(y, \bar{x})$ and $n(y, \bar{x})$ are pseudopolynomials in $y$;

P-2. $\operatorname{deg}_{y}\{m(y, \bar{x})\}<k ; \operatorname{deg}_{y}\{n(y, \bar{x})\} \leq 2 k-1$;

P-3. $\nu(m(y, \bar{x})) \geq k ; \nu(n(y, \bar{x}))>2 k$.

Let $n(y, \bar{x})=\sum_{i=0}^{p} n_{i}(\bar{x}) y^{p-i}$ (thus, $p \leq 2 k-1$ ). If $p<k$, by Lemma 4.5, $f(y, \bar{x})=\left(y^{k}+m(y, \bar{x})\right)^{2}+n(y, \bar{x})$ is a normal decomposition. Suppose that $p \geq k$. We have

$$
\begin{aligned}
f(y, \bar{x})=\left(y^{k}\right. & +\underbrace{m+\frac{1}{2} n_{0}(\bar{x}) y^{p-k}}_{m^{\prime}(y, \bar{x})})^{2} \\
& +\underbrace{\left(n-n_{0}(\bar{x}) y^{p}\right)-n_{0}(\bar{x}) m y^{p-k}-\frac{1}{4} n_{0}^{2}(\bar{x}) y^{2(p-k)}}_{n^{\prime}(y, \bar{x})} .
\end{aligned}
$$

\subsection{Lemma. In (4.4),}

P-1: $m^{\prime}(y, \bar{x})$ and $n^{\prime}(y, \bar{x})$ are pseudopolynomials in $y$;

P-2: $\operatorname{deg}_{y}\left\{m^{\prime}(y, \bar{x})\right\}<k ; \operatorname{deg}_{y}\left\{n^{\prime}(y, \bar{x})\right\} \leq p-1$;

P-3: $\nu\left(m^{\prime}(y, \bar{x})\right) \geq k ; \nu\left(n^{\prime}(y, \bar{x})\right)>2 k$.

Proof. By (P-1), and the definitions of $m^{\prime}(y, \bar{x})$ and $n^{\prime}(y, \bar{x}),(\mathrm{P}-1)$ is trivial.

Since $k<p \leq 2 k-1,(\mathrm{P}-2)$ follows immediately.

By $(\mathrm{P}-3), \nu\left(n_{0}(\bar{x}) y^{p-k}\right) \geq 2 k-k=k$ as required. Moreover,

$$
\exp \left\{n_{0}(\bar{x}) m(y, \bar{x}) y^{p-k}\right\}>\exp \left\{n_{0}(\bar{x}) y^{p}\right\}>\exp \left\{y^{2 k}\right\},
$$

which proves (P-3).

Proof of Lemma 4.6. Since $s(x) \neq 0$ and $t(x) \neq 0$ in the decomposition (4.1) of $q(x)$, we hence may assume that, after a suitable linear coordinate change,

$$
q(x)=\left(y^{2 k}+\sum_{i=1}^{2 k} \kappa_{i}(\bar{x}) y^{2 k-i}\right) \cdot i(x),
$$

where $i(x)$ is invertible, and each $\nu\left(\kappa_{i}\right) \geq i$ by the preparation theorem. Without loss of generality, we may assume that $i(x) \equiv 1$.

Starting with (4.5), we rewrite $q(x)$ as

$$
\begin{aligned}
q(x) & =y^{2 k}+y^{k}(\underbrace{\sum_{i=1}^{k} \kappa_{i}(\bar{x}) y^{k-i}}_{\alpha(y, x)})+\underbrace{\sum_{i=k+1}^{2 k} \kappa_{i}(\bar{x}) y^{2 k-i}}_{\beta(y, x)} \\
& =(y^{k}+\underbrace{\frac{1}{2} \alpha(y, \bar{x})}_{m(y, x)})^{2}+\underbrace{\beta(y, \bar{x})-\frac{1}{4} \alpha^{2}(y, \bar{x})}_{n(y, x)} \\
& =\left(y^{k}+m(y, \bar{x})\right)^{2}+n(y, \bar{x}) .
\end{aligned}
$$

Clearly, $m$ and $n$ are both pseudopolynomial in $y$ with $\operatorname{deg}_{y}\{m\}<k$ and $\operatorname{deg}_{y}\{n\} \leq 2 k-2$. Also, $\nu(m) \geq k$ and $\exp \{n\}>\exp \left\{\left(y^{k}+m\right)^{2}\right\}$. Thus, we 
may apply Lemma 4.8 if $\operatorname{deg}_{y} n \geq k$. Since $q(x)$ is not a completely squared element, we will get (4.3) after finitely many applications of Lemma 4.8.

Consider the function $q(x)$ and its normal decomposition (4.3) (in a suitable coordinate system). Let $\operatorname{in}(h(y, \bar{x}))=h_{0}(\bar{x}) y^{p}$. By the definition, $p \leq k-1$.

Write $s_{1}(y, \bar{x})=y^{k}+g(y, \bar{x})$, and $t_{1}(y, \bar{x})=h(y, \bar{x})$. We define a sequence $\left\{s_{i}(y, \bar{x})\right\}$ and $\left\{t_{i}(y, \bar{x})\right\}:$ For $i>1$,

$$
\begin{aligned}
& s_{i}(y, \bar{x})=s_{i-1}^{2}(y, \bar{x})+\frac{1}{2} t_{i-1}(y, \bar{x}) ; \\
& t_{i}(y, \bar{x})=-\frac{1}{4} t_{i-1}^{2}(y, \bar{x}) .
\end{aligned}
$$

The sequence satisfies

$$
s_{i-1}^{2} \ldots s_{1}^{2} \cdot q(y, \bar{x})=s_{i}^{2}(y, \bar{x})+t_{i}(y, \bar{x}), \quad i=1, \ldots .
$$

By the definition, we can see that

$$
\text { in }\left(s_{i}\right)=y^{2^{i-1} k}, \quad \text { in }\left(t_{i}\right)=h_{0}^{2^{i-1}}(\bar{x}) y^{2^{i-1} p} .
$$

Therefore, by Lemma 4.5, the expression (4.8) is a proper decomposition.

4.9. Lemma. Let $a(x) \in \mathbb{C}\{x\}$ with $\nu(a)>0$. Then,

$$
\tau\left\{a^{2} q(y, \bar{x})\right\} \leq 2(1+\tau\{q\}-\sigma\{q\}) \cdot \nu(a)+\sigma\{q\} .
$$

Proof. We may assume that $\operatorname{in}(a(x))=a_{0}(\bar{x}) y^{d}$. Take an integer $i$ such that $\log _{2} d+2 \geq i>\log _{2} d+1$. Consider

$$
a^{2} \cdot s_{i-1}^{2} \ldots s_{1}^{2} q(y, \bar{x})=a^{2} \cdot s_{i}^{2}(y, \bar{x})+a^{2} \cdot t_{i}(y, \bar{x}) .
$$

Clearly, $\operatorname{in}\left(a \cdot s_{i}(y, \bar{x})\right)=a_{0}(\bar{x}) y^{2^{i-1} k+d}$ and $\operatorname{in}\left(a^{2} t_{i}(y, \bar{x})\right)=a_{0}^{2} h_{0}^{2^{i-1}} y^{2^{i-1} p+2 d}$, by (4.9). By the choice of $i$, we have

$$
2^{i-1} k+d>2^{i-1} p+2 d \text {. }
$$

By Lemma 4.5, (4.11) is a proper decomposition of $a^{2} \cdot s_{i-1}^{2} \ldots s_{1}^{2} \cdot q(y, \bar{x})$. Let $a^{2} q(y, \bar{x})=u^{2}(y, \bar{x})+v(y, \bar{x})$ be a proper decomposition of $a^{2} q(y, \bar{x})$. Then $\nu\left(s_{i-1}^{2} \ldots s_{1}^{2} \cdot v(y, \bar{x})\right) \leq \nu\left(a^{2} t_{i}(y, \bar{x})\right)$. In other words,

$$
\left(2^{i-1}+\ldots+2\right) k+\tau\left\{a^{2} \cdot q(y, \bar{x})\right\} \leq 2 \nu(a)+2^{i-1} \tau\{q\},
$$

which gives

$$
\begin{aligned}
\tau\left\{a^{2} \cdot q(y, \bar{x})\right\} & \leq 2 \nu(a)+2^{i-1} \tau\{q\}-\left(2^{i}-2\right) k \\
& \leq 2(1+\tau\{q\}-\sigma\{q\}) \cdot \nu(a)+\sigma\{q\} .
\end{aligned}
$$

Remark. It should be pointed out that all the discussion here is valid if $q(x)$ and $a(x)$ are in $\mathbb{C} \llbracket x \rrbracket$.

4.2. Linear product estimates. In this section, we will show a linear product estimate for $(X, 0)$ which is defined by a pseudopolynomial of order 2 .

For an element $f=a(x) z+b(x)$ in $\mathscr{\sigma}_{X, 0}$, define $\gamma_{1}(x)$ and $\gamma_{2}(x)$ (also see (3.4) by the following relation:

$$
P_{f}(x, z)=\left(z-f\left(x, w_{1}(x)\right)\right)\left(z-f\left(x, w_{2}(x)\right)\right)=z^{2}+\gamma_{1}(x) z+\gamma_{2}(x) .
$$


4.10. Theorem. At $(X, 0)$,

$$
\nu\left(\gamma_{2}(x)\right) \leq 2(2+\tau\{q\}-\sigma\{q\}) \cdot \nu\left(\gamma_{1}(x)\right) .
$$

Proof. Let $f=a(x) z+b(x)$. By Property 1, we have to show the order of $\gamma_{2}$ is linearly bounded by the order of $\gamma_{1}$. In the case where $P(x, z)=z^{2}+q(x)$, we have $\gamma_{1}(x)=2 b(x)$ and $\gamma_{2}(x)=-a^{2}(x) q(x)+b^{2}(x)$.

If $2 \nmid \exp \{q(x)\}$ (i.e., $s(x) \equiv 0$ in the expressions of proper decomposition), we have

$$
\begin{aligned}
\nu\left(\gamma_{2}(x)\right) & =\nu\left(-a^{2}(x) q(x)+b^{2}(x)\right) \\
& =\min \{\nu(q(x))+2 \nu(a(x)), 2 \nu(b(x)) \leq 2 \nu(b(x))\} \\
& =2 \nu\left(\gamma_{1}(x)\right)
\end{aligned}
$$

If $2 \mid \exp \{q(x)\}$, then, we must have $s(x) \neq 0$ and $t(x) \neq 0$. By the discussion in $\S 4.2$, we may find a coordinate system and a normal form (4.3) of $q(x)$. We claim that for all $a(x)$ and $b(x)$,

$$
\nu\left(\gamma_{2}(x)\right)=\nu\left(-a^{2}(x) q(x)+b^{2}(x)\right) \leq \tau\left\{a^{2}(x) q(x)\right\} .
$$

In fact, if (4.13) is false, $a^{2}(x) q(x)=b^{2}(x)-\left(-a^{2}(x) q(x)+b^{2}(x)\right)$ which is also a decomposition of $a^{2}(x) q(x)$. That contradicts the definition of $\tau\left\{a^{2}(x) q(x)\right\}$.

Evidently, we need only to consider the case where $\nu\left(a^{2}(x) q(x)\right)=\nu\left(b^{2}(x)\right)$. By Lemma 4.9, we have

$$
\begin{aligned}
\nu\left(\gamma_{2}(x)\right) & \leq 2(1+\tau\{q\}-\sigma\{q\}) \nu(a)+\sigma\{q\} \\
& \leq 2 \cdot(2+\tau\{q\}-\sigma\{q\}) \cdot \nu\left(\gamma_{1}(x)\right) .
\end{aligned}
$$

Thus, (4.12) and (4.14) assure the linear product estimates.

4.3. Uniform linear product estimates. The discussion is actually local: we need to show uniform linear product estimates on any compact set containing 0 .

First, we give

4.11. Lemma. Let $\left(x^{\prime}, z^{\prime}\right) \in X$ and assume that the order of $P(x, z)$ at $\left(x^{\prime}, z^{\prime}\right)$ is 1 . Then, $P(x, z)$ is irreducible at $\left(x^{\prime}, z^{\prime}\right)$, and we may take $a=1$ and $b=0$ in the linear product estimate.

Proof. In this case, $\mathscr{O}_{X,\left(x^{\prime}, z^{\prime}\right)}$ is equivalent to $\mathbb{C}\{x\}$.

Thus, we need only to consider, in a neighborhood of 0 , that the closed analytic subset $Y \subset X$ on which $\nu(P(x, z))=2$. Clearly, by a proper choice of the coordinate $(x, z)$ (i.e., $p(x)=0), Y \subset\{(x, z) \mid z=0, \nu(q(x)) \geq 2\}$. Thus, we need to study $q(x)$ on the set $B=\{x \mid \nu(q(x)) \geq 2\} \cap U$, where $U$ is a relatively compact neighborhood of 0 .

Let $Z \subset X$ be analytic subsets. By $\mathscr{M}(X, Z)$ we mean the ring of meromorphic functions on $X$ with its poles in $Z$ (cf. [BM1, Chapter V]). An element in $\mathscr{M}(X, Z) \llbracket t_{1}, \ldots, t_{k} \rrbracket$ is called a parametrized family (with parameters in $X-Z$ ). For more discussion about parametrized families, see [BM1, Chapter V]. Let

$$
F_{x}\left(t_{1}, \ldots, t_{k}\right)=\sum_{\alpha} f_{\alpha}(x) t^{\alpha} \in \mathscr{M}(X, Z) \llbracket t_{1}, \ldots, t_{k} \rrbracket .
$$

For a point $p \in X-Z$,

$$
F_{p}\left(t_{1}, \ldots, t_{k}\right)=\sum_{\alpha} f_{\alpha}(p) t^{\alpha} \in \mathbb{K} \llbracket t_{1}, \ldots, t_{k} \rrbracket
$$


can be obtained by evaluating the coefficients $f_{\alpha}$ of the family at $p$.

There exists a finite stratification of $B$ by closed analytic subsets of $B$ :

$$
B=B_{0} \supset B_{1} \supset \ldots \supset B_{k}=\phi
$$

such that on each stratum $B_{i}-B_{i+1}$,

(1) $q(x)$ has a constant order, say $n_{i}$;

(2) there is a homeomorphism $x \rightarrow(\bar{x}, y)$, and parametrized families $d_{i, z}(\bar{x})$ $\in \mathscr{M}\left(B_{i}, B_{i+1}\right) \llbracket \bar{x} \rrbracket(i=1, \ldots, n)$ and $A_{i, z}(\bar{x}, y) \in \mathscr{M}\left(B_{i}, B_{i+1}\right) \llbracket \bar{x}, y \rrbracket$, such that at each point $z_{0} \in B_{i}-B_{i+1}$, the Taylor expansion of $q$ at $z_{0}$ is

$$
q_{z_{0}}(\bar{x}, y)=\left(y^{n_{i}}+\sum_{j=1}^{n_{i}} d_{j, z_{0}}(\bar{x}) y^{n_{i}-j}\right) \cdot A_{i, z_{0}}(\bar{x}, y),
$$

where $A_{i, z_{0}}(\bar{x}, y)$ is invertible, and $\operatorname{ord}\left(d_{j, z_{0}}(\bar{x})\right) \geq j$ (cf. [BM1, Chapter V]). Moreover, if $2 \mid n_{i}$, there exist $G_{i, z}(\bar{x}, y), H_{i, z}(\bar{x}, y)$ and $I_{i, z}(\bar{x}, y)$ in $\mathscr{M}\left(B_{i}, B_{i+1}\right) \llbracket \bar{x}, y \rrbracket$, such that

$$
g_{z_{0}}(\bar{x}, y)=\left(\left(y^{k}+G_{i, z_{0}}(\bar{x}, y)\right)^{2}+H_{i, z_{0}}(\bar{x}, y)\right) \cdot I_{i, z_{0}}(\bar{x}, y),
$$

and (4.16) is a normal decomposition of $q_{z_{0}}(\bar{x}, y)$ at any point $z_{0} \in B_{i}-B_{i+1}$.

4.12. Proposition. The function $z \rightarrow \tau\left\{q_{z}\right\}$ is locally constructible.

Proof. It suffices to show that $\tau\left\{q_{z}\right\}$ is constant on a union of differences of analytic subsets. For the stratification $\left\{B_{i}\right\}$ mentioned above, on each stratum, we have two cases:

1. If $2 \nmid n_{i}, \tau\left(q_{z}(\bar{x}, y)\right)=\operatorname{ord}\left(q_{z}\right)$ for all the point $z$ in $B_{i}-B_{i+1}$.

2. Otherwise, at any point $z \in B_{i}-B_{i+1}, \tau\left(q_{z}(\bar{x}, Y)\right)=\nu\left(H_{i, z}(\bar{x}, y)\right)$, as a consequence of Lemma 4.5. Then, the coefficients of $H_{i, z}$ are meromorphic functions on $B_{i}-B_{i+1}$ with all their poles outside $B_{i}-B_{i+1}$. Therefore, there is an upper bound on the order of $\tau\left(q_{z}\right)$ over $B_{i}-B_{i+1}$, as desired.

The proof of Theorem 4.1. Since $\sigma\{q\}$ is analytically semicontinuous on $X$ and $\tau\{q\}$ is locally constructible, both functions are locally uniformly bounded. By Property 2, the uniform linear product estimate follows.

\section{REFERENCES}

[BM1] E. Bierstone and P. Milman, The local geometry of analytic mappings, Dottorato di ricerca in matematica Universita di Pisa, Dipartimento di Matematica, 1988.

[BM2] _-, Relations among analytic functions, Ann. Inst. Fourier (Grenoble) 37:1, 37:2 (1987).

[BM3] _ The Newton diagram of an analytic morphism, and applications to differentiable functions, Bull. Amer. Math. Soc. 9 (1983).

[BM4] $\_$, Geometric and differential properties of subanalytic sets, Bull. Amer. Math. Soc. 25 (1991).

[BM5] _ _ Arc-analytic functions, Invent. Math. 101 (1990), 411-424.

[BM6] _ A simple constructive proof of canonical resolution of singularities, effective methods in algebraic geometry, Progress in Math. 94 (1991), 11-30.

[EH] P.M. Eakin and G.A. Harris, When $\Phi(f)$ convergent implies $f$ is convergent, Math. Ann. 229 (1977), 201-210.

[Ga] A. M. Gabrielov, Formal relations between analytic functions, Math. USSR Izv. 7 (1973), 1056-1088.

[Gl] G. Glaeser, Fonctions composées différentiales, Ann. of Math. 77 (1963), 193-209. 
[GR] H. Grauert and R. Remmert, Coherent analytic sheaves, Springer-Verlag, 1984.

[I1] S. Izumi, Linear complementary inequalities for orders of germs of analytic functions, Invent. Math. 65 (1982), 459-471.

[12] A measure of integrity for local analytic algebras, Publ. RIMS Kyoto Univ. 21 (1985), 719-735.

[13] Gabrielov's rank condition is equivalent to an inequality of reduced orders, Math. Ann. 276 (1986), 81-89.

[Ma] H. Matsumura, Commutative algebra, Benjamin/Cummings, Reading, Mass., 1980.

[Mi] P. Milman, Analytic and polynomial homomorphisms of analytic rings, Math. Ann. 232 (1978), 247-253.

[Na] M. Nagata, Local rings, Interscience, 1962.

[R1] D. Rees, Izumi's Theorem, commutative algebra, Springer-Verlag, 1989.

[R2] - Valuations associated with a local ring. I, Proc. London Math. Soc. 5 (1955), 107-128.

[R3] - Valuations associated with a local ring. II, Proc. London Math. Soc. 31 (1956), 228-235.

[T1] J-Cl. Tougeron, Idéaux de fonctions différentiables, Berlin-Heidelberg-New York, 1972.

[T2] - Sur les racines d'un polynome a coefficients series formells, real analytic and algebraic geometry, Lecture Notes in Math., vol. 1420, Springer-Verlag, 1990.

[W1] T. Wang, $A$ stratification given by Artin-Rees estimates, Canad. J. Math. 44:1 (1992).

[W2] Linear inequalities in local analytic geometry, Doctoral Dissertation, University of Toronto, 1992.

[Wh] H. Whitney, Complex analytic varieties, Addison-Wesley, 1972.

Department of Mathematics, University of Toronto, Toronto, Ontario M5S 1A1, CANADA

E-mail address: vangt@math.toronto.edu 